\title{
Transverse Schottky and beam transfer function measurements in space charge affected coasting ion beams
}

\author{
Stefan Paret, Vladimir Kornilov, and Oliver Boine-Frankenheim \\ FAIR Accelerator Theory, GSI Helmholtzzentrum für Schwerionenforschung GmbH, Planckstraße 1, 64291 Darmstadt, Germany
}

Thomas Weiland

TEMF, Technische Universität Darmstadt, Schlossgartenstraße 8, 64289 Darmstadt, Germany

(Received 17 August 2009; published 18 February 2010)

\begin{abstract}
Transverse Schottky spectra and beam transfer functions (BTFs) of coasting ion beams were measured in the heavy ion synchrotron SIS-18 in order to study the impact of space charge on the transverse beam dynamics. The particle number in the beam was varied to investigate the intensity dependence of the space-charge effect. No cooling was applied to the beams throughout the experiment. The expected deformation of the Schottky spectra and BTFs is observed. An analytic model with linear space charge is employed to describe the deformed Schottky and BTF signals. In this model, the incoherent space-charge force and the coherent forces due to impedances are treated separately. Using the model, the space-charge induced tune shift is evaluated both from the position and the form of the signals. The data are well described by the model, only in the high-intensity BTFs deviations are observed. The stability diagrams are shifted according to the space-charge parameter obtained from the BTFs. In addition, the tune shift is estimated by virtue of measured beam profiles and particle numbers. The estimated tune shift is of the same order of magnitude but smaller than the measured one. Possible explanations for deviations between the measurements, the model, and the estimation are discussed.
\end{abstract}

PACS numbers: 29.20.D-, 29.27.-a, 52.59.Sa, 41.75.Ak

\section{INTRODUCTION}

Collective effects often limit the intensity of charged particle beams in accelerators. After several decades of investigation [1,2], the theory of Landau damping and impedances can be found mostly in the literature (see e.g. $[3,4])$. However, the accurate prediction of stability limits and the cure of instabilities is still being investigated today due to the demand for increased beam intensities. Particularly the dynamics of space-charge dominated beams is still a subject of ongoing research, for instance in the framework of the projected FAIR synchrotrons at GSI $[5,6]$.

With transverse beam transfer functions (BTFs) and Schottky detection, two common tools for beam diagnostics are available for the investigation of beam dynamics with collective effects. Schottky measurements record the power spectrum of the beam. Statistical fluctuations of the beam current and its dipole moment give rise to bands in the Schottky spectrum [7]. It is routinely used to determine properties of low intensity beams, such as the incoherent tune. A nice feature of this technique is that it is nondestructive.

Measuring the same beam signal but while the beam is excited transversally and dividing the output by the excitation yields the BTF. It provides information on the beam properties and accelerator parameters similar to Schottky measurements, but with a better signal to noise ratio. In addition the BTF gives information about the stability of a beam [3]. The perturbation of the beam is usually negligible. In the heavy ion synchrotron SIS-18 [8] at GSI, the BTF technique has been applied to determine the momentum distribution and Landau damping characteristics [9].

The fact that these methods are sensitive to collective effects implies on the other hand, that for high beam currents the output of these tools cannot be interpreted properly without understanding the impact of highintensity effects. For instance, the tune cannot simply be read from the location of the maximum of a BTF or Schottky band if collective effects are present because they distort the signals $[3,7,10]$. This is, besides the beam stability, another concern for the operation of accelerators with intense beams.

One type of collective effects is caused by the transverse impedances of the accelerator environment. This can result in a coherent tune shift and may drive an instability. In contrary to impedances, space charge does not drive coherent instabilities. Nevertheless space charge is important for the stability characteristics, particularly of lowenergetic ion beams, because it reduces the individual particle tune. The resulting separation of the incoherent spectrum from the coherent line suppresses Landau damping and thus reduces the stability limits [11]. Still, the difference between the effects of impedances and space charge is only scarcely discussed in the literature.

Modeling beam dynamics with space charge is difficult because space charge exerts an incoherent nonlinear force on each particle. The shape of the force field depends on 
the transverse particle distribution. Hence, the analysis becomes quite involved, see, e.g., Ref. [6,12,13]—or simplifications must be made. One approach, which assumes a rigid beam, yields a dispersion relation allowing for space charge and impedances, as derived in Ref. [14]. Assuming a linear space-charge force, the dispersion relation was solved for a Gaussian momentum distribution in Ref. [15].

However, the assumed model is arguable because of the assumptions that the beam oscillates rigidly [16] and that the space-charge force is linear. On the other hand. in Ref. [6] self-consistent simulations are shown to compare well to a model based on a rigid beam. The nonlinear components of space charge are negligible for the weak to moderate space charge, as present during our measurement. In fact, measurements with a weakly relativistic ion beam (velocity over speed of light $\beta=0.15$ ), and particle tracking simulations demonstrate that the linear spacecharge model describes the shape of Schottky sidebands deformed by moderate space charge [10].

In this study, we report about a new experiment performed in SIS-18. BTFs and Schottky spectra, measured with the same settings, are compared to each other. The beam current was varied by more than 1 order of magnitude to study the dependence of space-charge effects on the beam intensity quantitatively. Compared to our previous measurements, a higher beam intensity was achieved. The space-charge parameters from the Schottky and BTF data are compared to an estimation based on measured beam parameters.

The diagnostic methods and the linear space-charge model are introduced in Sec. II. In Sec. III the realization of our measurement is described. The results and their comparison with the model follow in Sec. IV. Finally we conclude with a summary in Sec. V.

\section{SCHOTTKY AND TRANSVERSE BTF DIAGNOSTICS}

In synchrotrons and storage rings, Schottky diagnostics is commonly used for the measurement of the momentum distribution $\rho(p)$ of the particles that constitute the beam, the revolution frequency $f_{0}$, as well as the betatron tune $Q_{0}$. In the absence of collective effects, the shape of the Schottky bands reflects the momentum distribution [7]. The rms width of a longitudinal band centered around the revolution harmonic $m$ is given by $\sigma=$ $m f_{0}|\eta| \delta p / p$, where we introduced the rms momentum spread $\delta p / p$ and the slip factor $\eta=1 / \gamma_{T}^{2}-1 / \gamma^{2}$ with the transition point $\gamma_{T}$ and the Lorentz factor $\gamma$. Schottky sidebands $P_{0}(f)$ are located at

$$
f_{m, 0}^{ \pm}=f_{0}\left(m \pm Q_{f, 0}\right),
$$

where $Q_{f, 0}$ is the fractional part of $Q_{0}$. The "+" sign corresponds to the upper sideband and the "-" sign to the lower one. The rms width of $P_{0}$ reads

$$
\sigma_{m}^{ \pm}=\left|m \eta \pm\left(Q_{f, 0} \eta-Q_{0} \xi\right)\right| f_{0} \frac{\delta p}{p},
$$

where $\xi$ is the chromaticity. Using the short notation $z_{0}=$ $\left(f_{m, 0}^{ \pm}-f\right) / \sigma_{m}^{ \pm}$, the transverse BTF of a low intensity beam is given by [3]

$$
r_{0}\left(z_{0}\right)= \pm \int_{-\infty}^{\infty} \frac{P_{0}(z)}{z_{0}-z} d z .
$$

For a Gaussian $\rho$ the integration yields the complex error function [15]

$$
r_{0}\left(z_{0}\right)= \pm \sqrt{\frac{\pi}{2}} \frac{1}{\sigma} \mathrm{ie}^{-z_{0}^{2} / 2}\left(1-\frac{\operatorname{erf}\left(\mathrm{i} z_{0} / \sqrt{2}\right)}{\mathrm{i}}\right) .
$$

However, because the maximal momentum spread is actually limited to a finite value, a truncated Gaussian distribution could be more suitable to model $\rho$. Such a distribution complicates the analysis. Besides the fact that the integral in Eq. (3) can be solved only numerically, the problem is that the range of the momentum distribution is not accessible for measurements because of the signal to noise ratio. Therefore we assume an unlimited range in the following discussion.

In the following, $r$ denotes a BTF influenced by collective effects. The plot of the inverse BTF

$$
\frac{1}{r\left(z_{0}\right)}=U\left(z_{0}\right)+\mathrm{i} V\left(z_{0}\right)
$$

in the complex plane, where $U, V \in \mathbb{R}$, is called stability diagram [3]. First we consider an impedance $Z_{\perp}$, which lumps the geometric and material properties of the beam environment together [4]. The real part of $Z_{\perp}$ drives dipolar beam oscillations which eventually can lead to an instability. Imaginary impedances do not drive instabilities, but by virtue of the coherent tune shift $\Delta Q_{\text {coh }}=$ $r_{p} N Z^{2} /\left(2 \pi Z_{0} A Q_{0} \gamma\right) \operatorname{Im}\left(Z_{\perp}\right)$, they give rise to a gap between the coherent beam tune and incoherent particle tune, and hence suppress the transfer of energy from the coherent to the incoherent motion which is required for Landau damping $[4,11]$. Because of this gap, the actual particle density in the tails of $\rho(p)$ becomes particularly important for the effectiveness of Landau damping.

The deformation of both Schottky spectra and BTF due to $\Delta Q_{\text {coh }}$ is more conveniently expressed using the parameter $\Delta U_{\text {coh }}=\Delta Q_{\text {coh }} f_{0} / \sigma_{m}^{ \pm}$. Analogously, the impact of real impedances is described by the parameter $\Delta V$. A Schottky sideband deformed by an impedance is described by [7]

$$
P\left(z_{0}\right)=\frac{P_{0}\left(z_{0}\right)}{\left| \pm 1-\left(\Delta U_{\text {coh }}+\mathrm{i} \Delta V\right) r_{0}\left(z_{0}\right)\right|^{2}},
$$

and for transverse BTF we have [3]

$$
r\left(z_{0}\right)=\frac{r_{0}\left(z_{0}\right)}{ \pm 1-\left(\Delta U_{\mathrm{coh}}+\mathrm{i} \Delta V\right) r_{0}\left(z_{0}\right)} .
$$


The stability of a beam exposed to impedances can be displayed in a stability diagram which is given by the inverse of $r$ plotted in the complex plane [3]. The BTF on the upper and lower side of a harmonic yields two branches which enclose the area of stability. From Eqs. (5) and (7) follows

$$
\frac{1}{r\left(z_{0}\right)}= \pm \frac{1}{r_{0}\left(z_{0}\right)}-\left(\Delta U_{\mathrm{coh}}+\mathrm{i} \Delta V\right)
$$

which shows another feature of $\Delta U_{\text {coh }}+\mathrm{i} \Delta V_{\text {coh. }}$. It is a measure for the shift of the stability diagram due to an impedance. The beam becomes unstable when the origin is moved out of the area of stability.

Independently of the beam environment, the self-field, referred to as space charge, acts directly on each particle without affecting the coherent frequency. The force exerted on each particle depends on its position with respect to the beam center. Hence, space charge gives rise to an incoherent force, which depends on the transverse particle distribution. Since this force is nonlinear in real beams, particles with different amplitudes of the betatron oscillation are affected differently. As a consequence a tune spread occurs.

Assuming a rigidly oscillating beam, the equation of motion with space charge and impedances can be turned into a dispersion relation [14], which generally needs to be solved numerically. The problem is considerably simplified if a homogeneous beam profile, i.e., a KapchinskyVladimirsky (K-V) beam [17], is assumed. Space charge then becomes linear and the tune spread turns into an incoherent tune shift $\Delta Q_{\mathrm{sc}}$. Consequently, the fractional part of the incoherent tune becomes

$$
Q_{f}=Q_{f, 0}-\Delta Q_{\mathrm{sc}}
$$

and $f_{m, 0}^{ \pm}$[Eq. (1)] is shifted to

$$
f_{m}^{ \pm}=f_{0}\left(m \pm Q_{f}\right) .
$$

$Q_{f}$ can be directly calculated from $f_{m}^{ \pm}$. But as we will see below, it cannot be measured as easily as $f_{m, 0}^{ \pm}$at low intensity.

The dispersion relation with linear space charge is derived and solved for a Gaussian momentum distribution in Ref. [15]. Using the space-charge parameter

$$
\Delta U_{\mathrm{sc}}=\frac{\Delta Q_{\mathrm{sc}} f_{0}}{\sigma_{m}^{ \pm}}
$$

we put the dispersion relation from [15] together with Eq. (6) and (7) in order to model linear space charge. For a Schottky band this yields

$$
P(z)=\frac{P_{0}(z)}{\left| \pm 1-\left(\Delta U_{\mathrm{coh}}-\Delta U_{\mathrm{sc}}+\mathrm{i} \Delta V\right) r_{0}(z)\right|^{2}}
$$

and for the BTF

$$
r(z)=\frac{r_{0}(z)}{ \pm 1-\left(\Delta U_{\mathrm{coh}}-\Delta U_{\mathrm{sc}}+\mathrm{i} \Delta V\right) r_{0}(z)}
$$

with the normalized frequency

$$
z=\frac{1}{\sigma_{m}^{ \pm}}\left(f_{m}^{ \pm}-f\right) .
$$

Here we emphasize that $z$ incorporates $\Delta Q_{\mathrm{sc}}$ as opposed to $\Delta Q_{\text {coh. }}$. Note also that, due to their deformation, the maxima of $P(z)$ and $r(z)$ do not coincide with $f_{m}^{ \pm}$. According to Eq. (13), space charge shifts the transverse stability diagram analogously to an impedance. The shift from $z_{0}$ to $z$ is not visible in this representation. Therefore the shift of the stability diagrams in cold beams can be described in terms of a "space-charge impedance". This way the observations made with cooled beams have been discussed $[18,19]$.

Fitting Eq. (12) or Eq. (13) with a given $r_{0}$ to a set of measured data yields $\Delta U_{\mathrm{sc}}-\Delta U_{\text {inc }}$ from the shape of the signal. Hence, the deformation of the band tells the difference between a coherent and an incoherent tune. On the other hand, combining Eq. (14) and Eq. (10) produces $Q_{f}$. Comparison with the low intensity tune $Q_{f, 0}$ allows the determination of $\Delta Q_{\mathrm{sc}}$ [Eq. (9)] independently of $\Delta Q_{\mathrm{coh}}$ by virtue of

$$
\Delta Q_{\mathrm{sc}}=\frac{f_{m, 0}^{ \pm} \mp f_{m}^{ \pm}}{f_{0}}
$$

$\Delta Q_{\mathrm{sc}}$ in the $y$ plane can be estimated from $N$, the beam emittances $\epsilon_{x}, \epsilon_{y}$ and the mean beta functions $\beta_{x}, \beta_{y}$ by virtue of [20]

$$
\Delta Q_{\mathrm{sc}, y}=\frac{r_{\mathrm{p}} N Z^{2} g}{\pi \beta^{2} \gamma^{3} A\left(\epsilon_{y}+\sqrt{\epsilon_{y} \epsilon_{x} \beta_{x} / \beta_{y}}\right)},
$$

where $g$ is the form factor of the transverse density distribution. For a $\mathrm{K}-\mathrm{V}$ beam $g$ is 1 and $\epsilon$ is the full emittance. For a Gaussian distribution $g$ is 2 and $\epsilon$ the $2 \sigma$ emittance. In the latter case Eq. (16) yields the maximal one-particle tune shift while the incoherent tune of the beam is smeared out.

At the injection energy of SIS-18, corresponding to $\beta=$ 0.15 , the impact of impedances on the beam is expected to be negligible compared to space charge. One of the most important impedance sources is the beam pipe. For a perfectly conducting beam pipe of radius $b$ and for a beam radius $a$, the coherent tune shift is by a factor $a^{2} / b^{2}$ weaker than the space-charge tune shift [4]. During our experiment this factor was $\lesssim 0.1$, thus from here on we assume $\Delta Q_{\text {coh }} \approx 0$.

The most important real impedance stems from the resistive wall and the kickers. The former has been calculated analytically [21], the latter numerically [22]. For SIS18 and at the measuring frequency of about $10 \mathrm{MHz}$, the kicker impedance does not exceed $100 \mathrm{k} \Omega / \mathrm{m}$ and the resistive wall a fraction of it. For the most intense beam 
in our experiment this corresponds to $\Delta V \approx 3 \times 10^{-4}$. Consequently, real impedances were not relevant for the measurement and the deformation of the signals [Eq. (12) and (13)] is linked to $\Delta Q_{\text {sc }}$ only.

\section{EXPERIMENTAL SETUP}

In our measurement an uncooled coasting ${ }^{40} \mathrm{Ar}^{18+}$ beam was stored at the injection energy of $11.4 \mathrm{MeV} / \mathrm{u}$, corresponding to $\beta=0.15$ and $f_{0} \approx 214 \mathrm{kHz}$, in the synchrotron SIS-18 for several seconds. The number of ions $N$ stored in the ring was varied from $2.5 \times 10^{8}$ to $1.1 \times 10^{10}$. The lower limit was set by the sensitivity of the Schottky pickup and the upper by the beam stability on the injection plateau. In order to change $N$, the beam current in GSI's linear accelerator UNILAC [23] was adjusted. The parameters of the multiturn injection were fixed to prevent $\epsilon$ from changing.

The hardware for Schottky and BTF diagnostics in SIS18 is a replicate of the system designed for the experimental storage ring at GSI $[24,25]$. It comprises a plate capacitor as a pickup and a strip-line kicker to excite the beam for BTF measurements. The setup is sketched in Fig. 1. The signals from the two plates of the detector are preamplified and either added, to produce the longitudinal Schottky signal, or subtracted for transverse Schottky or BTF measurements. The pickup was operated in the nonresonant mode in our experiment, mainly because of the limited range of the resonance frequency.

On the injection plateau, either Schottky spectra were recorded or a network analyzer performed a frequency sweep to obtain BTFs around the harmonic $m=50$. The vertical plane was chosen because the smaller distance between the detector plates in this direction provides a better signal to noise ratio. In order to determine the revolution frequency and the momentum distribution,

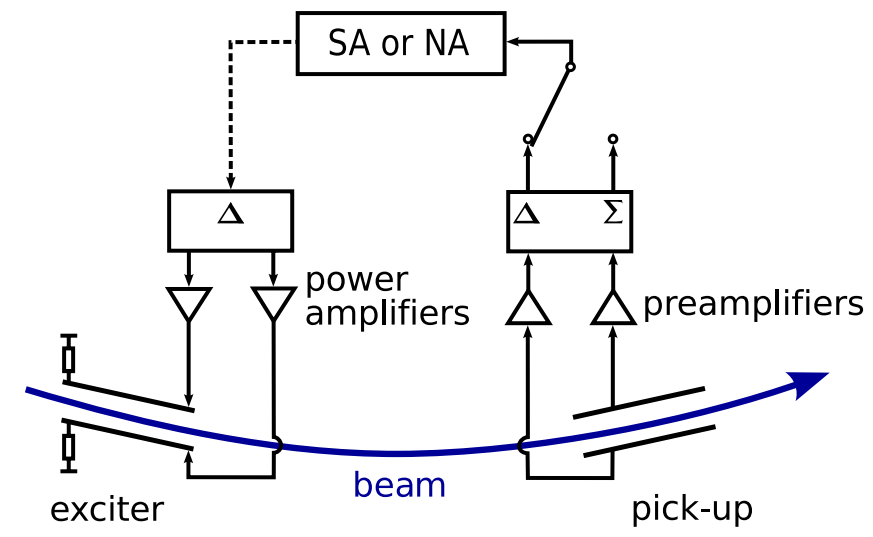

FIG. 1. (Color) The hardware for Schottky and BTF diagnostics. The dashed arrow refers to BTF measurements with a network analyzer (NA) only. In this case only the difference $(\Delta)$ signal from the detector is useful. For Schottky measurements, a spectrum analyzer (SA) is connected to the difference or sum $(\Sigma)$ signal of the pickup.
TABLE I. Measured particle number and momentum spread. In addition, the estimation of the space-charge tune-shift and the space-charge parameter are provided.

\begin{tabular}{lccc}
\hline \hline$N / 10^{9}$ & $\delta p / p / 10^{-4}$ & $\Delta Q_{\text {sc,est }}$ & $\Delta U_{\text {sc,est }}$ \\
\hline 0.25 & 2.5 & 0.001 & 0.09 \\
0.45 & 2.8 & 0.002 & 0.15 \\
0.90 & 4.2 & 0.004 & 0.19 \\
2.0 & 5.6 & 0.010 & 0.32 \\
3.9 & 6.7 & 0.019 & 0.53 \\
7. & 7.6 & 0.034 & 0.84 \\
10. & 7.8 & 0.048 & 1.2 \\
11. & 7.8 & 0.053 & 1.3 \\
\hline \hline
\end{tabular}

without being disturbed by collective effects, also longitudinal Schottky spectra were recorded. Since the beam was not cooled, the longitudinal phase-space density was too low for longitudinal space-charge effects.

The beam profile and the current were observed using an ionization profile monitor (IPM) and a transformer, respectively, in order to deduce $\epsilon$ and $N$. The vertical emittance was evaluated via $\epsilon_{y}=4 a_{y}^{2} / \beta_{y}$ with the rms beam width $a_{y}$ and the beta function $\beta_{y}$ at the position of the IPM. Here we applied the principle of rms equivalent beams [17] to approximate the full emittance of a K-V beam by the $2 \sigma$ emittance of the measured Gaussian beam profile. For the horizontal plane, we proceeded analogously.

The rms emittances were $\epsilon_{y} \approx 4.4 \mathrm{mmmrad}$ and $\epsilon_{x} \approx 6.0 \mathrm{mmmrad}$, with fluctuations of $0.3 \mathrm{~mm} \mathrm{mrad}$. With $\beta_{y}=11.8 \mathrm{~m}$ and $\beta_{x}=9.3 \mathrm{~m}$, the square root in Eq. (16) is well approximated by $\epsilon_{y}$-yielding the approximation for a circular beam. Using this approximation $\Delta Q_{\mathrm{sc}}$ was estimated. With the input of Eq. (2), an estimation of $\Delta U_{\mathrm{sc}}$ was obtained from Eq. (11). $N$ and $\delta p / p$ are listed in Table I with the estimated $\Delta Q_{\mathrm{sc}}$ and $\Delta U_{\mathrm{sc}}$.

\section{RESULTS}

The longitudinal Schottky spectra reveal a nearly Gaussian momentum distribution. They also indicate a dependency of the revolution frequency and the momentum spread on the injected beam current. This effect, though still being investigated, is explained by collective effects in the transfer channel from UNILAC to SIS-18. We will not discuss this issue here, but it should be noted that we compensated this frequency shift in order to make sure $f_{m}^{ \pm}$depends exclusively on $Q_{f}$. The data presented here were acquired at the lower sideband of the harmonic $m=50$.

Using Eq. (4), we fitted Eqs. (12) and (13) to the transverse spectra and BTFs, respectively, the latter by amplitude and phase, yielding $\Delta U_{\mathrm{sc}}$ from the shape and $Q_{f}$ from the position [using Eq. (10)]. Theoretically $\sigma_{m}^{ \pm}$could be obtained from the fit to the transverse spectra as well, but for strongly deformed signals the uncertainty of the fit 


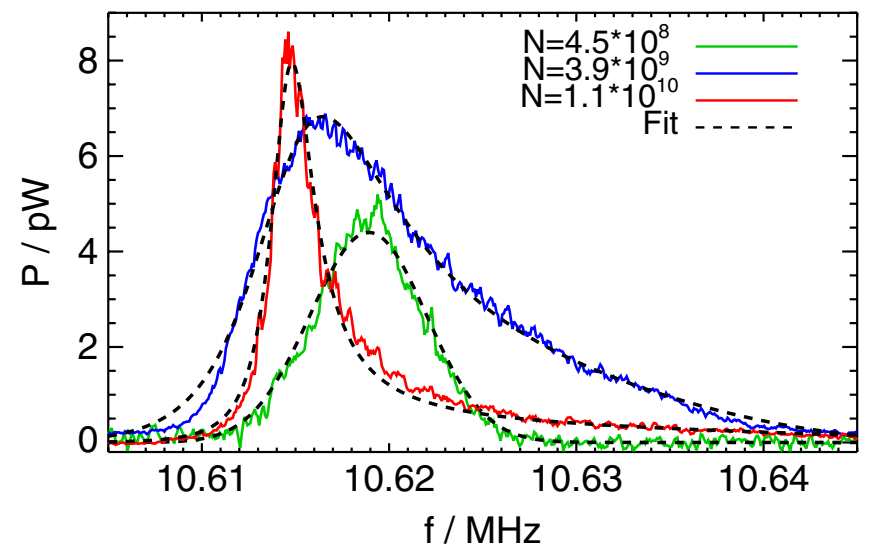

FIG. 2. (Color) Lower Schottky sideband at $m=50$ measured with different ion numbers and the corresponding fits. The corresponding $\Delta U_{\mathrm{sc}}$ are $0 ., 0.9$, and 1.8 , respectively. The curves are rescaled for a better prospect.

parameters turned out to be much lower when $\sigma_{m}^{ \pm}$was calculated using $\delta p / p$ from the longitudinal Schottky bands.

First we consider the shape of the signals. Below $N=$ $10^{9}$, where space charge is weak, the transverse Schottky bands and BTFs confirm the Gaussian momentum distribution. $\Delta U_{\mathrm{sc}}$ is zero with an uncertainty of \pm 0.2 .

The fit to the Schottky sidebands agrees excellently at all intensities. Figure 2 gives an example for three values of $N$. The BTFs on the other hand can be described only approximately as shown in Figs. 3 and 4. Particularly the spike in the phase cannot be reproduced by the model. Also the fit parameters of the amplitude and phase did not agree well at high intensity.

Stability diagrams constructed from the BTFs are shown in Fig. 5. One can see that they agree fairly well with the fit and that they are clearly shifted horizontally by space charge. From the fact that there is no vertical shift, we

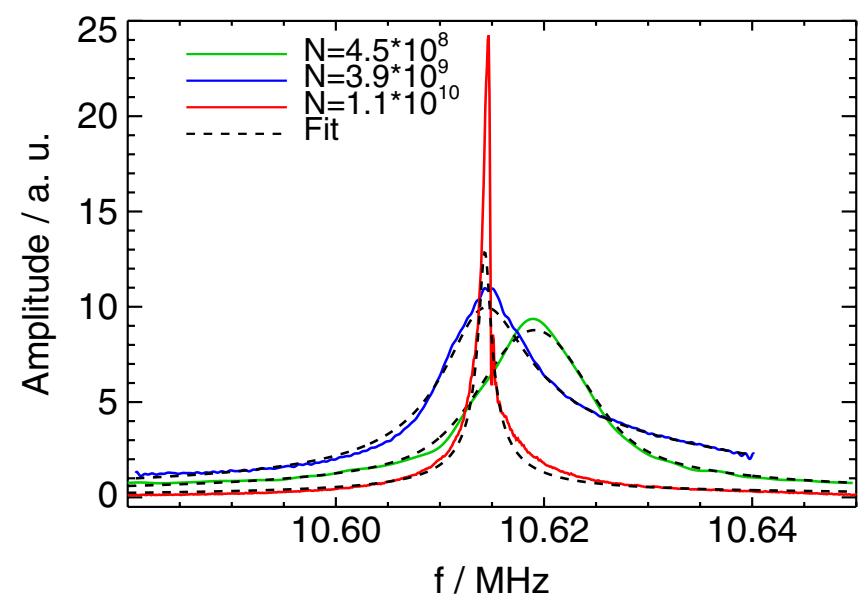

FIG. 3. (Color) Amplitude of the BTF for different currents. The settings are the same as in Fig. 2. $\Delta U_{\text {sc }}$ found for these data are $0 ., 0.9$, and 2.7. Also the fits are shown.

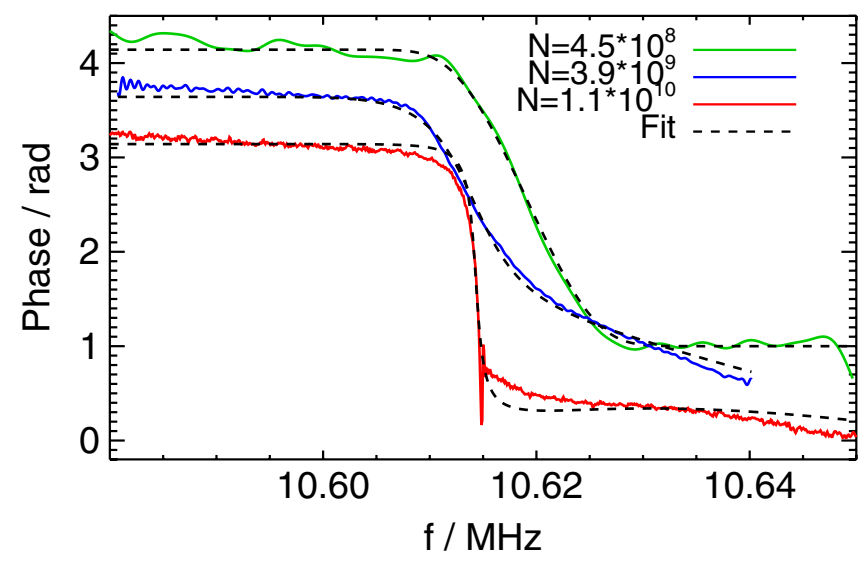

FIG. 4. (Color) BTF phases corresponding to the amplitudes shown in Fig. 3 with the fits. A vertical shift was added to separate the lines.

learn that no real impedance affected the beam to an observable extent.

A time gating procedure [26] was applied to suppress the noise on the BTF. This worked only with restrictions for the high-intensity data because of high order Fourier components which cannot be distinguished from noise. For that reason there is still noise on the red curve in the stability diagram (Fig. 5).

In a second step we compare the space-charge parameters and tune shifts quantitatively as functions of $N$. From the deformation we obtained $\Delta U_{\text {sc }}$. Alternatively $\Delta U_{\text {sc }}$ was extracted from the shift of $f_{m}^{ \pm}$applying Eqs. (15) and (11). $\Delta U_{\mathrm{sc}}$ obtained from Schottky and BTF measurements, as well as from the beam parameters, is shown in Fig. 6. The deviations of the measured $\Delta U_{\mathrm{sc}}$ reach up to \pm 0.5 with the BTF yielding slightly larger values than the Schottky spectra. For maximal intensity we found $\Delta U_{\mathrm{sc}} \approx 2$ from Schottky measurement and $\approx 2.5$ according to the BTF. The estimated space-charge parameter is significantly smaller.

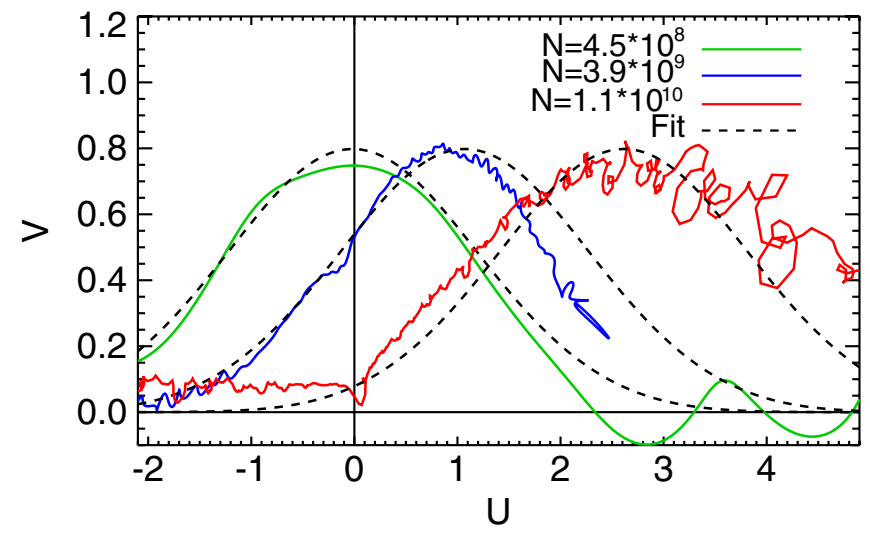

FIG. 5. (Color) Stability diagrams obtained from the BTF shown in Figs. 3 and 4. 


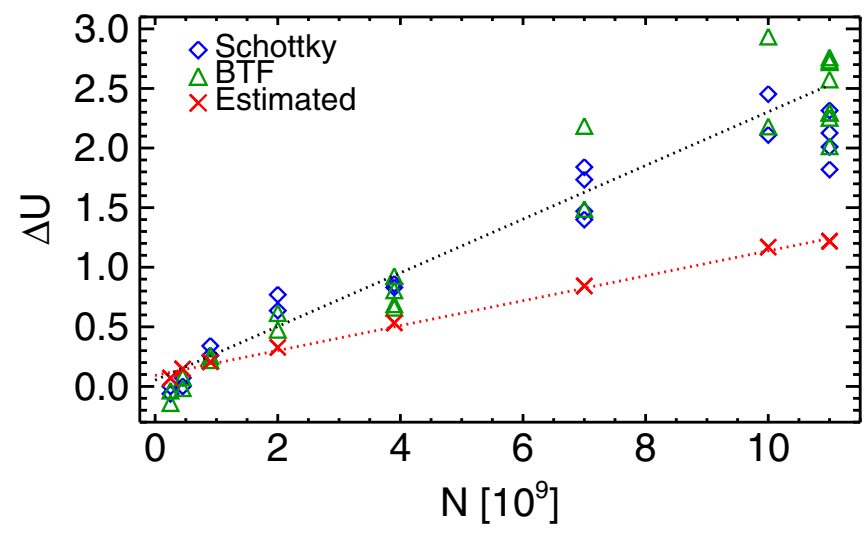

FIG. 6. (Color) Comparison of the space-charge parameters $\Delta U_{\text {sc }}$ obtained from Schottky (blue diamonds) and BTF (green triangles) measurements, and estimated from the beam parameters (red crosses) versus ion number. Least mean square fits to the estimated and measured points are shown, too.

The effective fractional tune $Q_{f}$ as obtained from $f_{m}^{ \pm}$is plotted in Fig. 7. In addition we show the estimated $\Delta Q_{\text {sc }}$ with respect to $Q_{f, 0}$. Also included is $Q_{f}$ plus $\Delta Q_{\mathrm{sc}}$ obtained from the deformation. As it nearly agrees with $Q_{f, 0}$, we confirmed that space charge is responsible for the tune shift. Again for the BTF (not plotted) larger discrepancies resulting from the mismatch between model and data were found.

The estimation from $N$ and $\epsilon$ predicts $\Delta Q_{\mathrm{sc}}$ to be about $40 \%$ smaller than the values detected via the Schottky measurements. We consider the Schottky data more accurate than the estimation, though there are some sources of uncertainty for the Schottky data as well. The important contributions are shot to shot fluctuations of injected beam current and particle loss during the measuring process. The observed changes of $N$ did not exceed $10 \%$.

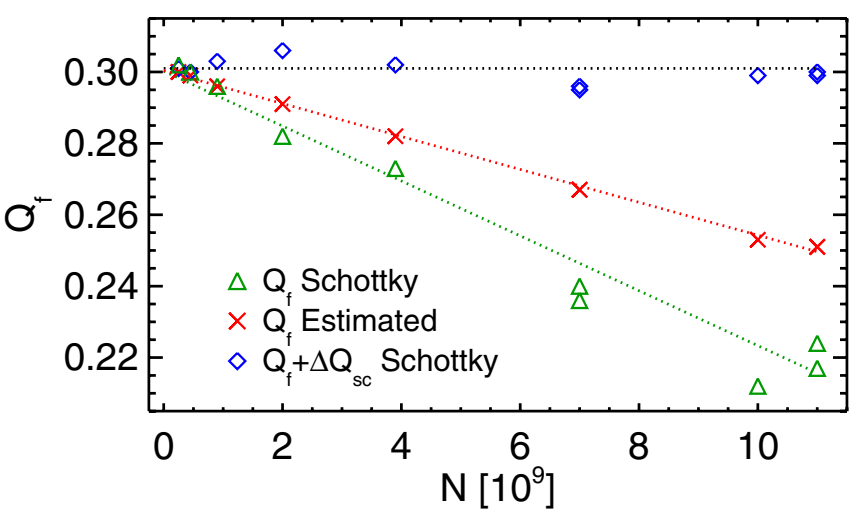

FIG. 7. (Color) Fractional part of the betatron tune $Q_{f}$ from the Schottky measurement (green triangles) and estimation (red crosses) for varying ion number. In addition, the reconstructed machine tune $Q_{f}+\Delta Q_{\mathrm{sc}}$ is plotted (blue diamonds). As it is always $\approx Q_{f, 0}$ (horizontal line), only space charge is responsible for the deformation. The nonhorizontal lines are least mean square fits to the effective tunes.
Also the momentum distribution in the tails may have been subject to fluctuations. As discussed in Sec. II the tails of the momentum distribution, which become more important for the deformed signals, cannot be measured. We know that the range of the distribution must be finite, but we have no information on the shape of the tails. Solving Eq. (3) numerically with a properly truncated Gaussian yields BTFs similar to the red curves in Figs. 3 and 4. But since the Schottky bands agree very well with the model this effect seems to be not very important. The reason for the lower degree of agreement between model and BTF is not understood. The excitation and damping process of a beam may be affected by space charge in a too complicated way to be described by our model.

The estimated space-charge parameters are mainly affected by the measuring error of $\epsilon$. The beta functions in the IPM were not measured but calculated with a beam optics code. The actual beta functions may deviate from the ideal ones as a consequence of closed orbit deformations [27]. The uncertainty of $a$, entering squared into $\epsilon$, is affected by the spacial resolution of the IPM of $2.1 \mathrm{~mm}$. Furthermore, the self-field of intense beams is known to falsify the output of the IPM [28]. A minor effect may be related to the modeling of the measured Gaussian beam profiles as K-V beam with $g=1$ [Eq. (16)]. Most importantly, the microchannel plates, a component of the IPM to magnify the signal strength, are known to degrade time after time, which may be responsible for a systematic measuring error.

Measurements were also done at the upper sideband. Similar results to the lower side were obtained, but with a larger uncertainty. The reason for this difference is not clear.

\section{SUMMARY}

An experiment dedicated to transverse Schottky and BTF measurements with space charge was performed with different beam currents. A model for linear space charge was employed to describe the Schottky spectra, BTFs, and stability diagrams of a coasting beam. The results are compared to an estimation based on measured beam parameters.

The Schottky spectra agree very well with the model up to the maximum intensity, corresponding to $\Delta U_{\mathrm{sc}} \approx 2$. The tune shift extracted from the deformation increases linearly with $N$. The tune shift according to the shift of the spectra is consistent with the parameter from the deformation. No evidence for a coherent tune shift was found. A discrepancy to the tune-shift estimation from the beam parameters was found. This observation is referred mainly to the uncertainty of the emittance. It is demonstrated that the analysis of transverse Schottky spectra with a linear space-charge model allows the determination of the incoherent tune of coasting beams with space charge. 
Also BTFs were measured at low and high intensity. Their deformation is described by the linear space charge rather well. The stability diagrams obtained from the BTF reproduce the shape expected due to the momentum distribution at all measured intensities. The observed intensity dependent shift of the stability diagram agrees with the model.

[1] L. J. Laslett, V. K. Neil, and A.M. Sessler, Rev. Sci. Instrum. 36, 436 (1965).

[2] J. Borer, G. Guignard, A. Hofmann, E. Peschardt, F. Sacherer, and B. Zotter, in Proceedings of PAC 1979 (IEEE, New York, 1979).

[3] A. W. Chao, Beam Physics and Accelerator Technology (Wiley, New York, 1993).

[4] H. Wiedemann, Particle Accelerator Physics, Nonlinear and Higher-Order Beam Dynamics (Springer, Berlin, 1995), Vol. 2.

[5] O. Boine-Frankenheim, I. Hofmann, and V. Kornilov, in Proceedings of the 10th European Particle Accelerator Conference, Edinburgh, Scotland, 2006, http://jacow.org/.

[6] V. Kornilov, O. Boine-Frankenheim, and I. Hofmann, Phys. Rev. ST Accel. Beams 11, 014201 (2008).

[7] S. Chattopadhyay, CERN Technical Report No. CERN 8411, 1984.

[8] K. Blasche and B. Franczak, in Proceedings of EPAC 1992 (Atlantica Séguier Frontières, Gif-sur-Yvette, 1992).

[9] V. Kornilov, O. Boine-Frankenheim, W. Kaufmann, and P. Moritz, GSI Technical Report No. GSI-Acc-Note-200612-001, 2006.

[10] O. Boine-Frankenheim, V. Kornilov, and S. Paret, Phys. Rev. ST Accel. Beams 11, 074202 (2008).

[11] A. Hofmann, in CAS: 5th General Accelerator Physics Course (CERN, Jyväskylä, Finland, 1994), 94-01_v1.
[12] M. Blaskiewicz, Phys. Rev. ST Accel. Beams 4, 044202 (2001).

[13] D. V. Pestrikov, Nucl. Instrum. Methods 578, 65 (2007).

[14] D. Möhl, CERN Technical Report No. CERN/PS 95-08 (DI), 1995.

[15] K. Y. Ng, Fermi National Accelerator Laboratory Technical Report No. FERMILAB-FN-0760-AD, 2004.

[16] A. Burov and V. Lebedev, Phys. Rev. ST Accel. Beams 12, 034201 (2009).

[17] M. Reiser, Beam Physics and Accelerator Technology (Wiley, New York, 1994).

[18] U. Oeftiger, Ph.D. thesis, Universität Bonn, 1994.

[19] U. Schaaf, K. Beckert, D. Budicin, H. Eickhoff, B. Franzke, I. Hofmann, G. Kalisch, F. Nolden, P. Spädtke, and M. Steck, in Proceedings of EPAC 1992 (Ref. [8]).

[20] K. Schindl, CERN Report No. CERN-PS-99-012-DI, 1999.

[21] A. M. Al-Khateeb, R. W. Hasse, O. Boine-Frankenheim, W. M. Daqa, and I. Hofmann, Phys. Rev. ST Accel. Beams 10, 064401 (2007).

[22] B. Doliwa, H. DeGersem, and T. Weiland, in Proceedings of the 21st Particle Accelerator Conference, Knoxville, 2005 (IEEE, Piscataway, NJ, 2005), p. 1820.

[23] W. Barth, in Proceedings of LINAC 2008, Victoria, BC, Canada, http://jacow.org/.

[24] K. Beckert, S. Cocher, B. Franzke, and U. Schaaf, in Proceedings of EPAC 1990 (Atlantica Séguier Frontières, Gif-sur-Yvette, 1990).

[25] U. Schaaf, Ph.D. thesis, Universität Frankfurt, 1991.

[26] F. Caspers, M. Chanel, and U. Oeftiger, in Proceedings of the Particle Accelerator Conference, Washington, DC, 1993 (IEEE, New York, 1993).

[27] A. Parfenove, G. Franchetti, and S. Sorge, GSI Technical Report No. ACC-note-2009-004, 2009.

[28] P. Forck, A. Bank, T. Giacomini, and A. Peters, in Proceedings of DIPAC 2005, Lyon, France, http:// jacow.org/. 